\title{
Improved Optical Properties of Sulphide based Core/Shell Nanostructures
}

\author{
M.Priya Dharshini, V.Shally, Sr. Gerardin Jayam*, S.Mary Delphine \\ Research Department of Physics, Holy Cross College (Autonomous), Nagercoil - 629 004, Tamil Nadu, India.
}

\begin{abstract}
Pure ZnS nanoparticles, $M n S / Z n S$ and $P b S / Z n S$ core/shell nanostructures are prepared by simple chemical method. UV studies reveal that the optical band gap increases for the samples in which the core with a small band gap is surrounded by the shell having a large band gap. There is a blue shift observed in all the samples. Comparing with the pure $\mathrm{ZnS}$ nanoparticles, $M n S / Z n S$ and PbS/ZnS core/shell nanostructures, $P L$ studies show that there is slightly enhanced blue emission and an additional green emission.
\end{abstract}

Subject classification: PACS: 61.46.-w, 78.67.-n,81.07.-b,81.07.Wx.

"Corresponding author : e.mail: gerardin2312@yahoo.in

\section{Introduction}

Nanostructures can be obtained in metallic, ceramic, semi-conducting and diamond materials. The nanopowder industry combines nanotechnology and many applications of nanostructured materials. One important research area is the formation of semiconductor "quantum dots". There are two different types of nanostructures: "free" nanoparticles, where at some stage in production or use individual nanoparticles of a substance are present; Nanocomposites, nanostructured surfaces and nanocomponents (electronic, optical, sensors etc.), where nanoscale particles are incorporated into a substance, material or device ("fixed" nanoparticles). These free nanoparticles could be nanoscale species of elements, or simple compounds, but also complex compounds where for instance a nanoparticle of a particular element is coated with another substance ("coated" nanoparticle or "core-shell" nanoparticle) [1]. Some quantum dots are small regions of one material buried in another with a larger band gap. These can be so-called core-shell structures, e.g., with CdSe in the core and $\mathrm{ZnS}$ in the shell.

Nanoscale semiconductor materials doped with impurities as activators have been the subject of an extensive research interest in recent years due to their applications for a variety of commercial devices [2-6]. As one of well-known II-VI compound semiconductors, $\mathrm{ZnS}$ is particularly suitable for use as a luminescent host material for a variety of dopants due to its wide band gap energy at room temperature [7]. The self-organization of nanostructures across extended length scales is a key challenge in the design of integrated materials with advanced functions for various nanotechnology applications [8-12]. Self-assembly of nanocrystals provides an elegant bottom-up approach for the preparation of extended, highly ordered nanostructures with highly useful properties [13]. Zinc sulphide is utilized in a wide range of applications, e.g. photocatalysts, photoconductors, optical sensors, optical coatings, electro-optic modulators, field effect transistors, electroluminescent materials, solid-state solar window layers, light-emitting materials, etc [14].

It can be doped as both n-type semiconductor and p-type semiconductor, which is unusual for the II-VI semiconductors. Both sphalerite and wurtzite are intrinsic, wide-bandgap semiconductors. The cubic form has a band gap of $3.72 \mathrm{eV}$ whereas the hexagonal form has a band gap of $3.77 \mathrm{eV}$. $\mathrm{ZnS}$ is particularly suitable for use as luminescent host materials for a variety of dopants due to its wide band gap energy at room temperature [15]. One can easily achieve photoluminescence (PL) at different wavelengths by doping $\mathrm{ZnS}$ with different activating metal ions. Among them, $\mathrm{Mn}$-doped $\mathrm{ZnS}$ nanocrystals have been given much more research attention, mainly because of their promising applications. Unfortunately, a large portion of atoms are located on or near surfaces because of their high surface-to-volume ratio arising from the small particle size. This causes the surface states to act as luminescent quenching centers, resulting in low luminescence efficiency. In order to conquer such serious shortcomings, accordingly, core/shell structural nanomaterials have been developed and shown dramatically enhanced properties. Enhanced luminescence has been observed in $\mathrm{ZnS}: \mathrm{Mn} / \mathrm{ZnS}$ nanocrystals, ZnS:Mn/ZnO nanoparticles, and ZnS:Mn/ nanocrystals [14]. Daixun Jiang et al. [14] synthesized $\mathrm{Mn}$-doped $\mathrm{ZnS}$ nanorods by solvothermal method and were successfully coated with $\mathrm{ZnS}$ shells of various thicknesses. The powder X-ray diffraction (XRD) measurements showed the ZnS:Mn nanorods were wurtzite structure the $\mathrm{ZnS}$ shells formed from small particles. Room temperature photoluminescence (PL) spectra showed that the intensity of $\mathrm{Mn}$ emission first increased and then decreased with the thickening of the $\mathrm{ZnS}$ shells.

$\mathrm{PbS}$ is an indirect band gap semiconductor with a bulk band gap of about $0.41 \mathrm{eV}$. It has smaller effective masses of charge carriers, high value of dielectric constant, and a fairly high value of excitonic size. 
For these reasons it allows strong quantum confinement even in relatively large $\mathrm{PbS}$ quantum dots. Lead sulfide crystallizes in the sodium chloride motif, unlike many other IV-VI semiconductors.

Interest in PbS-based nanomaterials has been aroused because of their unique optical and emission properties, which have tremendous applications in lasers, light-emitting devices, detectors, nonlinear optics, single electron devices, optical switches, optical amplification, telecommunication, etc. $\mathrm{PbS}$ is interfaced with $\mathrm{ZnS}$ i.e., is added to $\mathrm{PbS}$. The luminescent properties of $\mathrm{ZnS}$ are enhanced. The electronic and emission behavior of the composite $\mathrm{PbS} / \mathrm{ZnS}$ particles is very different compared to either pure $\mathrm{ZnS}$ and $\mathrm{PbS}$ nanoparticles or their additive behavior: the optical, photophysical, and electronic properties of these nanoparticles can be manipulated by changing the surface environment, which may have important implications in the areas of photonics and photophysics [16]. Recent advances in the design and control of heterostructures and superlattices in 1D nanoscale semiconductors have opened the door to new device concepts. Onedimensional heterostructures consisting of two or even more important functional materials are of prime importance for revealing unique properties. They are also essential for developing potential nanoelectronic and optoelectronic devices [17].

A. V. Lukashin et al. [18] synthesized PbS/amorphous S core - shell nanoparticles in the LDH (Layered Double Hydroxide) matrix with the use of only one stage of photochemical modification of the $\mathrm{Mg}-\mathrm{Al}$ LDH with intercalated ions. The effect of the shell on the optical properties of lead sulfide is most likely associated with the surface modification of the PbS nanoparticles. Anil Kumar et al. [16] reported that the surface of $\mathrm{ZnS}$ and $\mathrm{PbS}$ has been modified by interfacing $\mathrm{PbS}$ on $\mathrm{ZnS}$ and $\mathrm{ZnS}$ on $\mathrm{PbS}$ nanoparticles. This produced core-shell nanocomposites $\mathrm{ZnS} / \mathrm{PbS}$ and $\mathrm{PbS} / \mathrm{ZnS}$ with tunable electronic properties. Jian Xu et al. [19] reported the growth of PbS shells over colloidal PbSe nanocrystal quantum dots (NQDs) with monolayer-precision. The technique of successive ion layer adsorption and reaction (SILAR) has been adapted to the growth of high-quality core-shell nanocrystals and the photoluminescence efficiency of the surfaceengineered core-shell nanoparticles was substantially higher than that of the plain core structures undergoing the same surface processing. Anil Kumar et al. [20] reported the deposition of $\mathrm{ZnS}$ layer as shell at the interface of $\mathrm{ZnS} / \mathrm{PbS}$ produces $\mathrm{ZnS} / \mathrm{PbS} / \mathrm{ZnS}$ nanocomposite. The electronic properties of the nanocomposite have been examined as a function of thickness of $\mathrm{ZnS}$ shell and in the presence of excess $\left[\mathrm{Zn}^{2+}\right]$. The monolayer of $\mathrm{ZnS}$ shell red shifts the onset of absorption of precursor $\mathrm{ZnS} / \mathrm{PbS}$ accompanied with an increase in absorption coefficient in the wavelength range of $200-600 \mathrm{~nm}$ having an excitonic absorption at $280 \mathrm{~nm}$. These particles display relatively intense blue-shifted $505 \mathrm{~nm}(2.45 \mathrm{eV})$ emission associated with a tremendous enhancement of fluorescence lifetime to $150 \mathrm{~ns}$ from $5.5 \mathrm{~ns}$, observed for precursor.

In this paper, the optical properties of $\mathrm{ZnS}$ nanoparticles, $\mathrm{MnS} / \mathrm{ZnS}$ and $\mathrm{PbS} / \mathrm{ZnS}$ core/shell nanocomposites is reported.

\subsection{Synthesis of $\mathrm{ZnS}$ nanoparticles}

\section{Experimental Section}

$0.125 \mathrm{M}$ zinc acetate and $0.125 \mathrm{M}$ sodium sulphide were dissolved separately in double distilled water and stirred constantly using magnetic stirrer. Both the solutions were mixed together and stirred thoroughly. To this solution, $0.2 \mathrm{M} \mathrm{NaOH}$ was added drop by drop to maintain a $\mathrm{pH}$ of 12 . Then the solution was placed on the hot plate and heated for about $80{ }^{\circ} \mathrm{C}$. After cooling to room temperature the precipitate thus obtained was filtered and washed out for 10 times using double distilled water. The sample was dried to obtain fine powder of pure $\mathrm{ZnS}$ nanoparticles.

\subsection{Synthesis of $\mathrm{MnS} / \mathrm{ZnS}$ core/shell nanostructures}

$0.09 \mathrm{~g}$ of the as-prepared $\mathrm{Mn}$ doped $\mathrm{ZnS}$ sample is well dispersed in $12.5 \mathrm{ml}$ of double distilled water using ultrasonicator for about 1 hour 15 minutes. Then, $25 \mathrm{ml}$ of $0.125 \mathrm{M}$ zinc acetate is added dropwise, while stirring. 10 minutes later, $25 \mathrm{ml}$ of $0.125 \mathrm{M}$ thiourea is added dropwise to the solution. After 10 minutes, $0.1 \mathrm{M}$ $\mathrm{NaOH}$ is added drop by drop to maintain a $\mathrm{pH}$ of 12 . Then the solution is heated in microwave oven for about $80^{\circ} \mathrm{C}$. The solution is then allowed to reach the room temperature and the precipitate thus obtained is filtered and washed out for 10 times using double distilled water and dried in the hot air oven at $60^{\circ} \mathrm{C}$ for 5 hours. The dried sample is crushed as fine powder in agate mortar and used for characterization purposes.

\subsection{Preparation of $\mathrm{PbS} / \mathrm{ZnS}$ core/shell nanostructures}

$0.19 \mathrm{~g}$ of the as-prepared $\mathrm{Pb}$ doped $\mathrm{ZnS}$ sample is well dispersed in $12.5 \mathrm{ml}$ of double distilled water using ultrasonicator for about 1 hour 15 minutes. $25 \mathrm{ml}$ of zinc acetate is added dropwise, while stirring. 10 minutes later, $25 \mathrm{ml}$ of thiourea is added dropwise to the solution. After 10 minutes, $\mathrm{NaOH}$ is added to maintain a pH of 12. Then the solution is heated for about $90^{\circ} \mathrm{C}$ using microwave oven. The solution is then allowed to reach the room temperature and the precipitate thus obtained is filtered and washed out for 10 times using 
double distilled water and dried in the microwave oven at $100^{\circ} \mathrm{C}$ for 3 hours. The dried sample is crushed as fine powder in agate mortar and used for characterization purposes.

\subsection{Characterization}

UV-VIS-NIR spectra of the the pure $\mathrm{ZnS}$ nanoparticles, $\mathrm{MnS} / \mathrm{ZnS}$ and $\mathrm{PbS} / \mathrm{ZnS}$ core/shell nanostructures are taken in the wavelength between $190 \mathrm{~nm}$ and $1100 \mathrm{~nm}$. The spectrometer is initialized with glass plate and the sample is mounted for recording absorption. The photoluminescence Spectroscopy for the prepared samples is carried out using Cary Eclipse Fluorescence Spectrophotometer at a scan rate of 600 $\mathrm{nm} / \mathrm{min}$.

\subsection{UV studies}

\section{Result And Discussion}

The optical absorbance of the pure $\mathrm{ZnS}$, nanoparticles are as shown in Fig.1, Fig.2, and Fig.3 respectively. From Fig.1, it can be seen that the strongest absorption peak of the as-prepared samples appears at around $319 \mathrm{~nm}$, which is fairly blue-shifted from the absorption edge of the bulk $\mathrm{ZnS}(345 \mathrm{~nm})$. This is due to the quantum confinement effect in the nanoparticles. Similar results are observed by Borah et al. [21], Rema Devi et al. [22]. ZnS has good absorption for light in the wavelength of 220-350 nm [22].

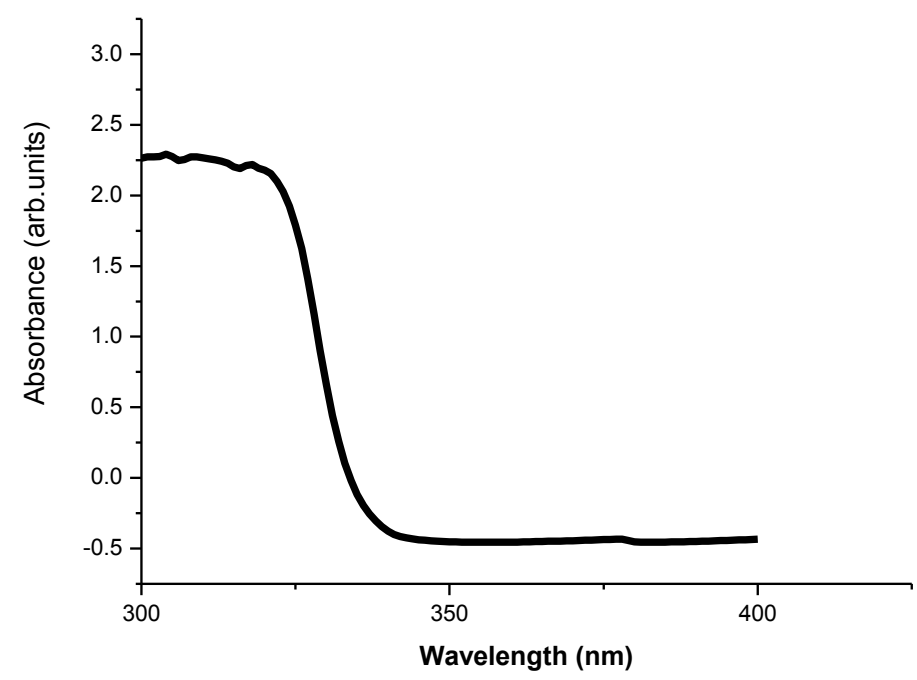

Fig.1. Optical absorbance spectrum of pure ZnS Nanoparticles

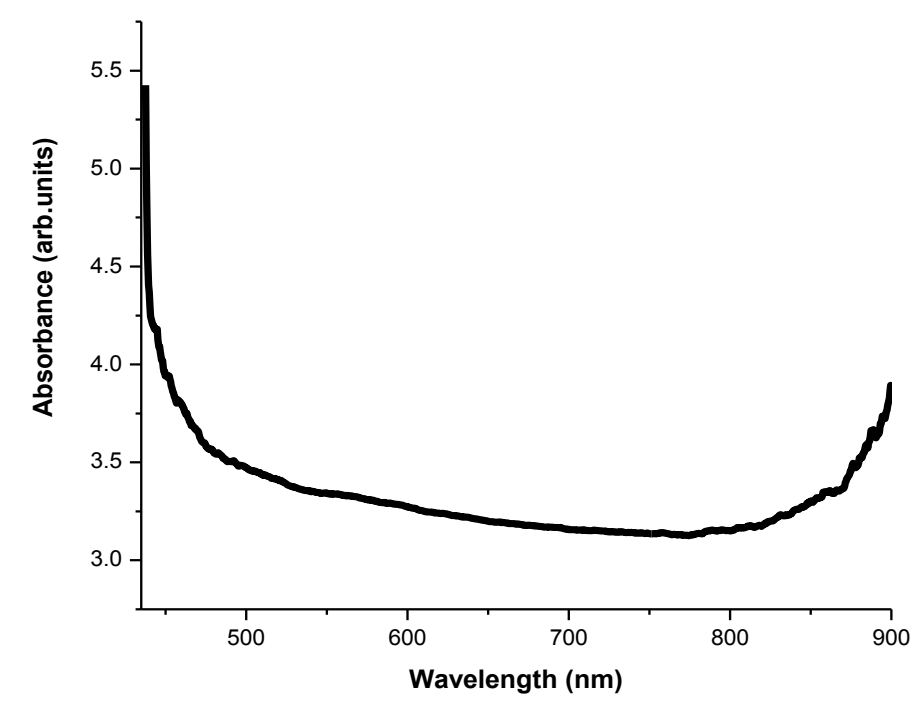

Fig.2. Optical absorbance spectrum for $\mathrm{MnS} / \mathrm{ZnS}$ core/shell nanostructures 


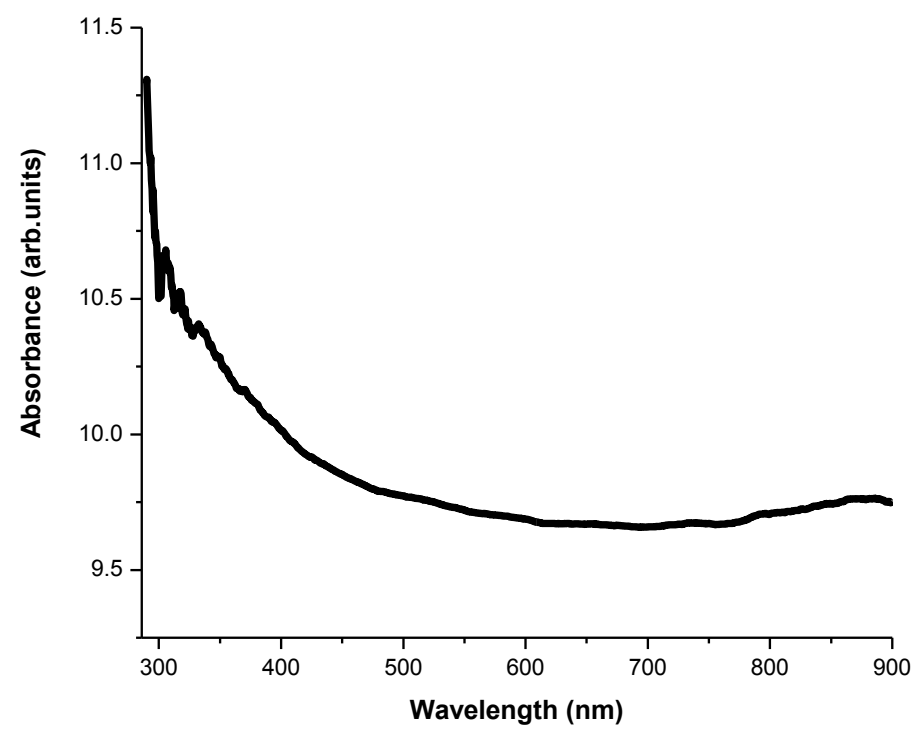

Fig.3. Optical absorbance spectrum for $\mathrm{PbS} / \mathrm{ZnS}$ core/shell nanostructures

The MnS/ZnS sample shows maximum absorbance of 5.41739 at a wavelength of $439 \mathrm{~nm}$. The absorbance decrease as the wavelength increases and attains 3.2956 at a wavelength of $852 \mathrm{~nm}$.

The $\mathrm{PbS} / \mathrm{ZnS}$ sample shows maximum absorbance of 11.2996 at a wavelength of $289 \mathrm{~nm}$. The absorbance decrease as the wavelength increases and attains 9.7587 at a wavelength of $927 \mathrm{~nm}$. In all these samples, the maximum absorbance shifts to the lower wavelength region.

Fig.4, Fig.5 and Fig.6 show the curves of ( $\alpha$ hv) ${ }^{2}$ versus photon energy (hv) for the $\mathrm{ZnS}, \mathrm{MnS} / \mathrm{ZnS}$ and $\mathrm{PbS} / \mathrm{ZnS}$ core/shell nanostructures respectively.

The optical band gap energy is determined by the intercept of the extrapolation to zero with photon energy axis $(\alpha h v)^{1 / 2} \rightarrow 0$. Table.1 shows the optical band gap of the samples. The band gap is increased for the $\mathrm{MnS} / \mathrm{ZnS}$ sample. Similar increase in optical band gap is observed for the $\mathrm{PbS} / \mathrm{ZnS}$ sample. This is because in $\mathrm{MnS} / \mathrm{ZnS}$ and $\mathrm{PbS} / \mathrm{ZnS}$ samples, the material having smaller band gap acts as the core. Also the charge carriers in the $\mathrm{MnS}$ and $\mathrm{PbS}$ are trapped by the shell i.e., $\mathrm{ZnS}$.

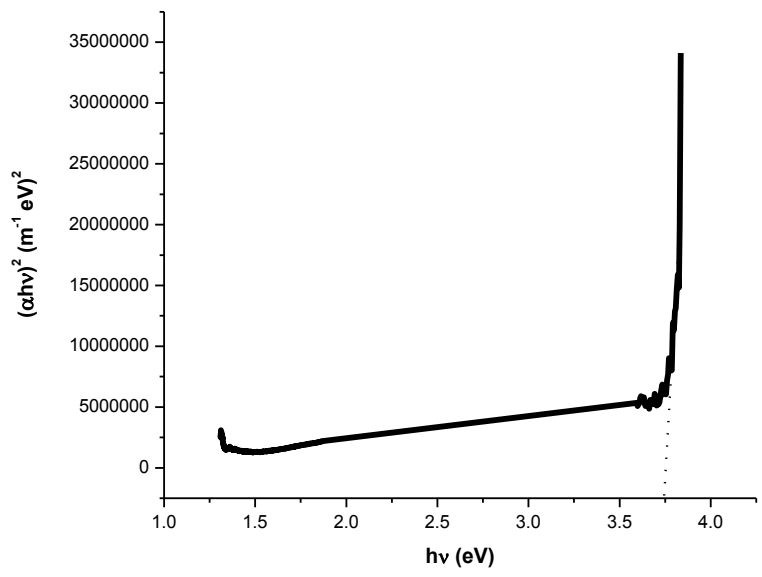

Fig.4.Tauc Plot of $\mathrm{ZnS}$ nanoparticles 


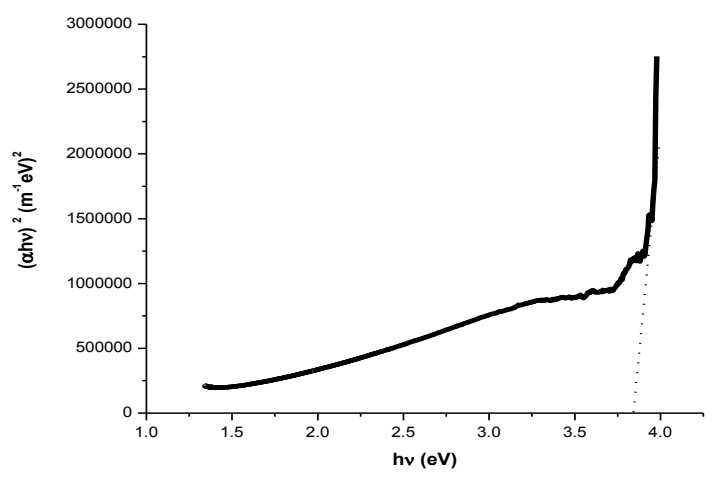

Fig.5. Tauc Plot of MnS/ZnS core/shell nanostructures

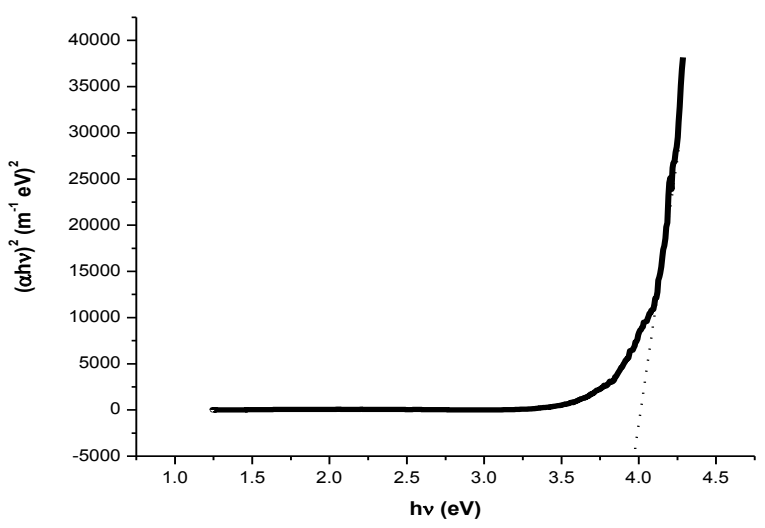

Fig.6.Tauc Plot of $\mathrm{PbS} / \mathrm{ZnS}$ core/shell nanostructures

Table 1 - Optical band gap of the as-prepared samples

\begin{tabular}{|l|l|l|}
\hline S.No. & Sample & Optical band gap(eV) \\
\hline 1. & $\mathrm{ZnS}$ & 3.7317 \\
\hline 2. & $\mathrm{MnS} / \mathrm{ZnS}$ & 3.8399 \\
\hline 3. & $\mathrm{PbS} / \mathrm{ZnS}$ & 3.9681 \\
\hline
\end{tabular}

\subsection{Photoluminescence Studies}

Fig.7, Fig.8 and Fig.9 depict the room temperature photoluminescence (PL) emission spectra for the pure $\mathrm{ZnS}$ nanoparticles, $\mathrm{MnS} / \mathrm{ZnS}$ and $\mathrm{PbS} / \mathrm{ZnS}$ core/shell nanostructures respectively. All the samples were subjected to an excitation wavelength of $260 \mathrm{~nm}$. All these PL emission plots contain two peaks centered around $360 \mathrm{~nm}$ and $419 \mathrm{~nm}$ approximately.

Since the particles sizes are very small, the size effect generated emission bands exhibit blue shift accordingly [12]. In the PL process, an electron from the $\mathrm{ZnS}$ valence band is excited across the band gap and the photoexcited electron subsequently decays by a normal recombination process to some surface or defect states [2].

The appearance of a strong blue emission at around $419 \mathrm{~nm}$ is attributed to the presence of sulphur vacancies in the lattice [2,23]. Similar results are reported by Namrata et al. [24]. This emission results from the recombination of photogenerated charge carriers in shallow traps $[25,26]$. The other peak exhibits a weak UV emission centered around $360 \mathrm{~nm}$ is due to the band to band transition of nanocrystalline $\mathrm{ZnS}$.

Observation of weak PL intensity at lower wavelength in the undoped $\mathrm{ZnS}$ samples indicates that there were insufficient luminescent centers available to modify the electronic structure of particles and yield the optimum and high fluorescence intensities. The lowering of PL intensity was attributed to concentration quenching; this effect is known to increase further for higher doping concentrations [27]. 


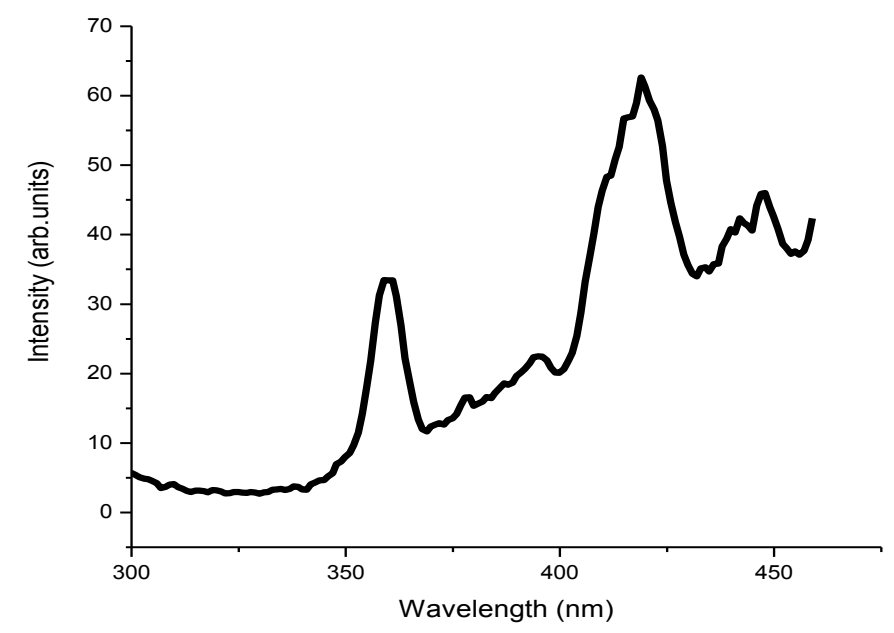

Fig.7. PL Emission spectrum of pure $\mathrm{ZnS}$ nanoparticles at an excitation wavelength of 260nm

In the case of $\mathrm{MnS} / \mathrm{ZnS}$ core/shell nanoparticles, there is slightly enhanced blue emission and an additional green emission at $360 \mathrm{~nm}$ and $494 \mathrm{~nm}$ respectively. The enhancement in the emission is due to the fact that high band gap $\mathrm{ZnS}$ shell material suppresses the tunneling of the charge carriers from the MnS core to the surface atoms of the shell, resulting in more photogenerated electrons and holes confined inside the $\mathrm{MnS}$ cores. Consequently, passivated nonradiative recombination sites that exist on the core surfaces lead to high PL efficiency $[16,20]$. The emission of peak around $\approx 360 \mathrm{~nm}$ can be ascribed to the hole traps originating from the unsaturated $s p^{3}$ orbital of the surface $\mathrm{S}$ atoms. The emission of peak around $\approx 515 \mathrm{~nm}$ has been tentatively ascribed to surface states or either the self-activated defect centre related to the zinc vacancies or the transfer energy between the energy level of sulfur vacancies and zinc vacancies [28].

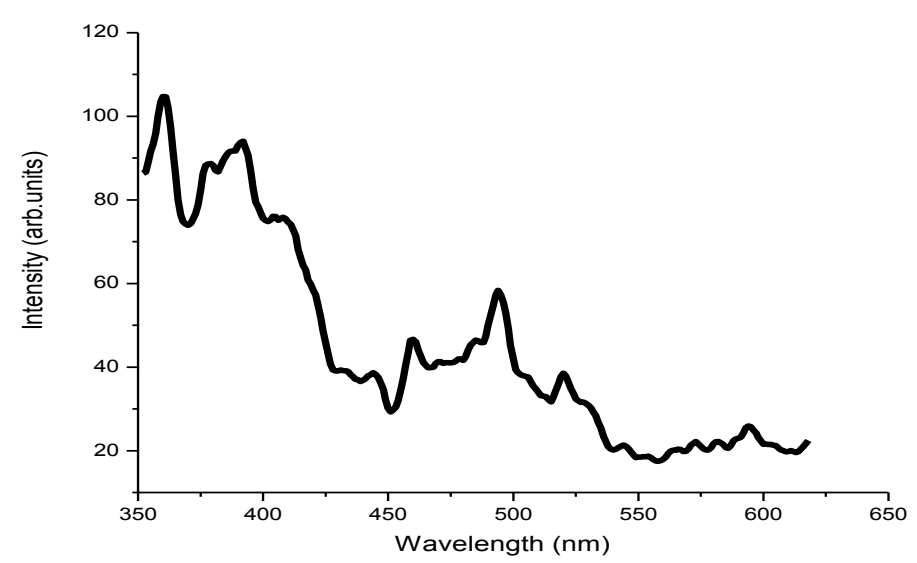

Fig.8. PL Emission spectrum of $\mathrm{MnS} / \mathrm{ZnS}$ core/shell nanostructures at an Excitation wavelength of 260nm 


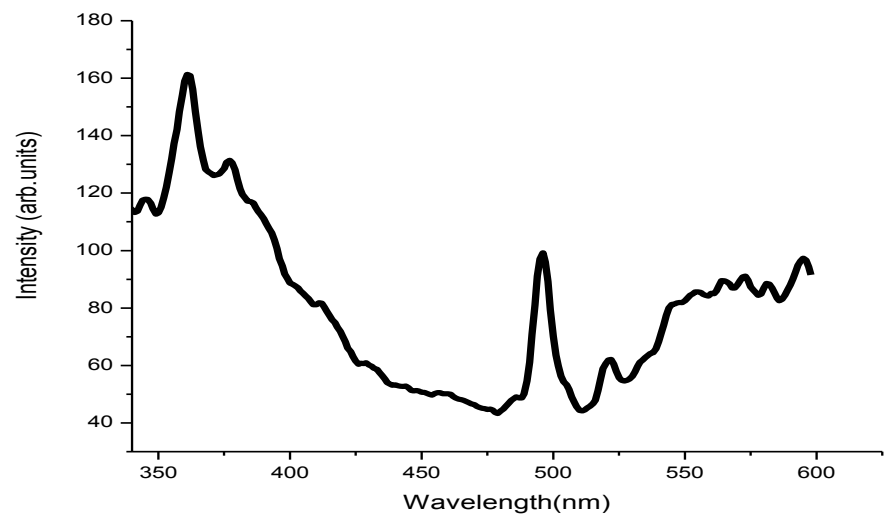

Fig.9. PL Emission spectrum of $\mathrm{PbS} / \mathrm{ZnS}$ core/shell nanocomposites at an excitation wavelength of $260 \mathrm{~nm}$

When compared to the PL emission spectra of $\mathrm{ZnS}$ sample, the $\mathrm{PbS} / \mathrm{ZnS}$ sample at the same excitation wavelength exhibits a strong blue emission at $360 \mathrm{~nm}$ and a green emission at $495 \mathrm{~nm}$. It is thus apparent that the optical, photophysical, and electronic properties of these nanoparticles can be manipulated by changing the surface environment, which may have important implications in the areas of photonics and photophysics [19].

\section{Summary And Conclusion}

The optical properties of the prepared samples pure $\mathrm{ZnS}$ nanoparticles, $\mathrm{MnS} / \mathrm{ZnS}$ and $\mathrm{PbS} / \mathrm{ZnS}$ core/shell nanostructures using UV-Vis-NIR Spectroscopy is studied. The optical band gap increases for the samples in which the core with a small band gap is surrounded by the shell having a large band gap. The Photoluminescence Spectroscopic studies reveal that there is a blue emission and a green emission in all the as-prepared samples. There is slight enhancement in the emission of $\mathrm{MnS} / \mathrm{ZnS}$ and $\mathrm{PbS} / \mathrm{ZnS}$ samples. The enhancement in the emission is due to the fact that high band gap $\mathrm{ZnS}$ shell material suppresses the tunneling of the charge carriers from the $\mathrm{MnS}$ and $\mathrm{PbS}$ cores to the surface atoms of the shell. The photoluminescence studies proved that the $\mathrm{MnS} / \mathrm{ZnS}$ core/shell nanocomposites exhibited excellent optical properties showing prominent blue and green emission. Hence they are promising to be used as high-performance visible-light-driven photocatalyst in future.

\section{Acknowledgement}

The authors gratefully acknowledge UGC for the financial support from the UGC XI plan -Minor Research Project No: F. MRP - 3827/11 (MRP/UGC-SERO).

\section{References}

[1]. S.Gopalachandra, Dictionary of Nano-Science and Nano-Technology, Alfa Publications, New Delhi, (2006), 195-205.

[2]. Karar.N, Singh.F, Mehta. B.R, J Appl phys., 95, (2004), 656.

[3]. Cruz AB, Shen Q, Toyoda T, Mater Sci Eng C, 25, (2005), 761

[4]. Ding Y, Wang XD, Wang ZL, Chem Phys Lett., 398, (2004), 32.

[5]. Tang W, Cameron DC, Thin Solid Films, 280, (1996), 221.

[6]. Bredol M; Merikhi J, J Mater Sci., 33, (1998), 471

[7]. Chen W, Malm JO, Zwiller V, Huang Y, Liu S, Wallenberg R, Bovin JO, Samuelson L Phys Rev B, 61, (2000), 11021.

[8]. H.Colfen, S. Mann, Angew. Chem., Int.Ed., 42, (2003), 2350.

[9]. Y.Xia, P.Yang, Y.Sun, Y.Wu, B.Mayers, B.Gates, Y.Yin, F.Kim, Adv. Mater., 15, (2003), 353.

[10]. S.I. Stupp, V. LeBonheur, K. Walker, L.S.Li, K.E.Huggins, M.Kesser, A. Amstutz, Science, 276, (1997), 384

[11]. C.B. Murray, C.R. Kagan, M.G. Bawaendi, Science, 270, (1995), 1335.

[12]. A.P. Alivisatos, Adv. Mater., 10, (1998), 1297.

[13]. A.K. Boal, F. Ilea, J.E. Derouchey, T. Thurn-Albrecht, T.P. Russell, V.M. Rotello, Nature, 204, (2000), 404.

[14]. Daixun Jiang, Lixin Cao,Ge Su,Wei Liu ,Hua Qu, Yuanguang Sun, Bohua Dong, J Mater Sci, 44, (2009), 2792-2795.

[15]. Xiaosheng Fang, Yoshio Bando, Ujjal K. Gautam, Tianyou Zhai, Haibo Zeng, Xijin Xu, Meiyong Liao and Dmitri Golberg, Critical Reviews in Solid State and Materials Sciences, 34 (3), (2009), 190- 223

[16]. Anil Kumar, Anshuman Jakhmola; Journal of Colloid and Interface Science, 297, (2006), 607-617.

[17]. Fei Li, Xueqin Liu, Tao Kong, Zhen Li, and Xintang Huang ,Cryst. Res. Technol., 44(4), (2009), 402 - 408.

[18]. A. V. Lukashin, A. A. Eliseev, N. G. Zhuravleva, S. V. Kalinin, A. A. Vergetel, and Academician Yu. D. Tret’yakov; $\square \square$ Doklady Chemistry, 383(4-6), (2002), 93-96.

[19]. Jian Xu, Dehu Cui, Ting Zhu, Gary Paradee, Ziqi Liang, Qing Wang, Shengyong Xu and Andrew Y Wang, Nanotechnology, 17, (2006), 5428.

[20]. Anil Kumar, Anshuman Jakhmola and Vidhi Chaudhary; Journal of Photochemistry and Photobiology A: Chemistry, 208(2-3), (2009), 195-202.

[21]. Jyoti P.Borah, J.Barman, K.C.Sarma, Chalcogenide Letters, 5(9), (2008), 201 -208

[22]. B.S.Rema Devi, R.Raveendran and A.V.Vaidyan, Pramana - Journal of Physics, 68(4), (2007), 679-687.

[23]. I. Yu, T. Isobe, M. Senna, J.Phys. Chem. Solids, 57, (1996), 373.

[24]. Namrata Dixit, Hemant Soni, Mukesh Chawda and Dhanajay Bodas, Materials Letters, 63(30), (2009), $2669-2671$.

[25]. Introduction to nanoscale science and technology edited by Massimiliano Di Ventra, Stephane Evoy, James R. Heflin, Jr., Springer Science + Business Media LLC, Reprinted (2009), 1.

[26]. R.N.Bhargava, D.Gallangher, T.Welker, J. Luminescence, 60, (1991), 275

[27]. Ding Y, Wang XD, Wang ZL, Chem Phys Lett., 398, (2004), 32.

[28]. J. Li, G.J.Fang, C.Li, L.Y.Yuan, L.Ai, N.S.Liu, D.S.Zhao, K.K. Ding, G.H.Li, X.Z.Zhao, Appl. Phys. A, 90, (2008), 759-763. 